\title{
Cellular function of satellite cells does not play a role in muscle weakness of adult Ts1Cje mice
}

Chai Ling Lim ${ }^{1}$, Usman Bala ${ }^{1,2}$, Melody Pui-Yee Leong ${ }^{3}$, Johnson Stanslas ${ }^{4}$, Rajesh Ramasamy ${ }^{5,6}$, King-Hwa Ling ${ }^{3,6}$ and Pike-See Cheah ${ }^{1,6,{ }^{*}}$

${ }^{1}$ Department of Human Anatomy, Faculty of Medicine and Health Sciences, Universiti Putra Malaysia, 43400 UPM Serdang, Selangor, Malaysia.

${ }^{2}$ Department of Human Anatomy, College of Medical Sciences, Gombe State University, Gombe, Nigeria.

${ }^{3}$ Department of Biomedical Science, Faculty of Medicine and Health Sciences, Universiti Putra Malaysia, 43400 UPM Serdang, Selangor, Malaysia.

${ }^{4}$ Department of Medicine, Faculty of Medicine and Health Sciences, Universiti Putra Malaysia, 43400 UPM Serdang, Selangor, Malaysia.

${ }^{5}$ Department of Pathology, Faculty of Medicine and Health Sciences, Universiti Putra Malaysia, 43400 UPM Serdang, Selangor, Malaysia.

${ }^{6}$ Genetics and Regenerative Medicine Research Centre, Faculty of Medicine and Health Sciences, Universiti Putra Malaysia, 43400 UPM Serdang, Selangor, Malaysia.

* Correspondence: cheahpikesee@upm.edu.my; Tel.: +603-8947-2355

Received: 10 March 2018; Accepted: 11 May 2018; Published: 15 May 2018

Edited by: Noraishah Mydin Abdul-Aziz (University of Malaya, Malaysia)

Reviewed by: Thilakavathy Karuppiah (Universiti Putra Malaysia, Malaysia); Meow-Keong Thong (University of Malaya, Malaysia)

DOI: https://doi.org/10.31117/neuroscirn.v1i1.6

\begin{abstract}
Down syndrome (DS) is a genetic condition resulting from triplication of human chromosome (HSA)21. Besides intellectual disability, DS is frequently associated with hypotonia. Satellite cells are the resident cells that provides robust and remarkable regenerative capacity to the skeletal muscles, and its population size has been reported to be disease-associated. However, little is known about the population size of satellite cells in DS and the association of its intrinsic cellular functionality and hypotonia seen in DS. Here, we studied the Ts1Cje mouse, a DS murine model displays the muscle weakness characteristic. Satellite cell populations were immunostained with Pax7 and myonuclei numbers in the Ts1Cje extensor digitorum longus muscle were assessed. Their cellular function was further determined via in vitro assay in high-serum conditioned medium. Subsequently, the in vitro self-renewal, proliferative, and differentiation activities of these myogenic precursor cells were assessed after 24, 48, and 72h using Pax7, MyoD, and Ki67 immunomarkers. Our results showed that the population and functionality of Ts1Cje satellite cell did not differ significantly when compared to the wildtype cells isolated from disomic littermates. In conclusion, our findings indicated that intrinsic cellular functionality of the satellite cells, do not contribute to muscle weakness in Ts1Cje mouse.
\end{abstract}


Keywords: Down syndrome; skeletal muscle; stem cells; muscle weakness; satellite cells

C2018 by Lim et al for use and distribution in accord with the Creative Commons Attribution (CC BY-NC 4.0) license (https://creativecommons.org/licenses/by-nc/4.0/), which permits unrestricted non-commercial use, distribution, and reproduction in any medium, provided the original author and source are credited.

\section{INTRODUCTION}

Down syndrome (DS) is a chromosomal condition caused by trisomy of human chromosome 21 (HSA21), which occurs at a rate of approximately 1 in every 700 live births worldwide [1-ㄹ․ The extra copy of chromosome 21 caused the imbalance expression of HSA21 and non-HSA21 genes, and eventually leads to various phenotypic features in DS individuals such as cognitive impairment, craniofacial abnormalities and hypotonia.

Hypotonia is a state of low muscle tone accompanied by a slower response speed together with reduced muscle strength [ $\underline{3}-\underline{4}]$. Cowley et al. []ㅡ reported that the force generated by the knee extensor muscle of DS individuals is approximately $40 \%-70 \%$ less than that generated by individuals with intellectual disabilities other than DS and those with normal intellectual ability.

Satellite cells are rare mononuclear cells with a low cytoplasmic content that are wedged between the basal lamina and sarcolemma of postnatal skeletal muscle [ 6 ]. In adult skeletal muscle, satellite cells are mitotically quiescent under normal circumstances and become activated in response to exercise and muscle injury [7]. Activated satellite cells, identified by their expression of myogenic regulatory factor MyoD, transiently re-enter the cell cycle to proliferate and subsequently exit the cell cycle to differentiate or self-renew []ㅡ. Hence, satellite cells play an essential role in skeletal muscle regeneration and maintenance of homeostasis [9]. It is hypothesized that a small defect in satellite cells can lead to a series of complications in recurrent regeneration. Many studies have reported that impairment of satellite cells is responsible for the pathogenesis of many muscular diseases [10-11]. Unfortunately, only limited information on the population size of the satellite cells in DS skeletal muscle and in exploring their role in muscle weakness seen in animal models of DS.

In this study, Ts1Cje mouse model of DS, also known as $\mathrm{T}(12 ; 16) 1 \mathrm{Cje}$ [12] was used to identify possible mechanisms involved in DS-associated hypotonia, focusing on characterization of the population size and cellular function of satellite cells isolated from Ts1Cje mouse skeletal muscles. The Ts1Cje mouse displays a remarkable number of phenotypic characteristics reminiscent of those commonly observed among DS individuals, including structural and cognitive alterations of the brain, craniofacial alterations and congenital heart defects [13]. Recent studies reported that Ts1Cje display reduced grip strength and locomotor activity compared to disomic/wildtype (WT) littermates [14]. Thus, Ts1Cje mice have considerable value in studies investigating the mechanisms of DS-associated pathology.

\section{MATERIALS AND METHODS}

\subsection{Animals and ethics statement}

This study was approved by the Institutional Animal Care and Use Committee (IACUC), Universiti Putra Malaysia (UPM) (Reference number: UPM/IACUC/AUPR003/2014). Adult disomic C57BL/6 mice (WT/control) and adult trisomic Ts1Cje mice at postnatal days (P) 5670 were used. They were generated by mating male Ts1Cje mice with female C57BL/6 mice, and their genotypes were determined by tail genotyping as described previously $[\underline{12}, \underline{15}]$. All mice were bred under controlled environmental conditions with a 12-h light/12-h dark cycle at $21-23^{\circ} \mathrm{C}$ and $55 \%$ relative humidity. Food and water were available ad libitum with a standard pellet diet. All mice were euthanized by cervical dislocation after being anesthetized in a chamber containing $2.5 \%$ isoflurane in $100 \%$ oxygen. 


\subsection{Single myofiber isolation, immunostaining, and analysis}

The extensor digitorum longus (EDL) muscle from adult Ts1Cje mice ( $n=9 ; 4$ males, 5 females) and age-matched control littermates $(n=9 ; 4$ males, 5 females) were harvested using a tendon-to-tendon approach. Single myofibers were isolated as described previously [16]. The satellite cells were immunostained with Pax7 (1:5; Developmental Studies Hybridoma Bank, lowa City, USA), while the myonuclei were counterstained with 4, 6-diamidino-2-phenylindole (DAPI). Subsequently, fluorescence microscopic images were acquired using an Olympus BX51 fluorescence microscope (Olympus, Tokyo, Japan) equipped with an UPlanF1 objective lens with Olympus ANALYSIS ${ }^{\circledR}$ FIVE acquisition software. Further image processing was achieved using ImageJ software (http://rsbweb.nih.gov/ij/). Satellite cells and myonuclei were counted manually in images captured at 10X magnification with the aid of ImageJ to determine the number of each myofiber. Student's ttest was performed to test for significant differences between the two genotypes. This experiment was conducted on 20 isolated EDL myofibers per replicate and biological triplicates for each genotype (e.g., a total of $\geq 80$ collected myofibers per genotype).

\subsection{Primary myoblasts isolation, culture, immunostaining and analysis}

The EDL of six adult male mice ( $\mathrm{n}=3$ per genotype) was harvested and digested in $0.2 \%(\mathrm{w} / \mathrm{v})$ collagenase type I solution at $37^{\circ} \mathrm{C}$ for $60 \mathrm{~min}$. The cells were pelleted by centrifuged at $300 \mathrm{~g}$ for 5 minutes at $16^{\circ} \mathrm{C}$. The cell pellet was resuspended in $0.05 \%$ Trypsin-EDTA (Gibco, Paisley, UK), and serial trituration was subsequently performed using pipettes with different bore sizes (from 10-ml serological pipette, $5-\mathrm{ml}$ serological pipette, 1-ml pipette tip to $18 \mathrm{G}$ needle attached to a 1-ml syringe). The satellite cell-trypsin mixture was then incubated at $37^{\circ} \mathrm{C}$ for $10 \mathrm{~min}$, centrifuged at $300 \mathrm{~g}$ for $10 \mathrm{~min}$ at $16^{\circ} \mathrm{C}$. The cell pellet was resuspended in conditioned medium [DMEM containing sodium pyruvate, $20 \%$ fetal bovine serum (Gibco), 10\% horse serum (Hyclone, Logan, UT, USA), $1 \%$ chick embryonic extract (Gemini Bio Products, West Sacramento, CA, USA), and $1 \%$ penicillin- streptomycin (Gibco)] and filtered through a 40- $\mu \mathrm{m}$ nylon cell strainer (Corning Incorporated, Corning, NY, USA). The cell suspension was preplated in uncoated T75 tissue culture flask (Techno Plastic Products, Trasadingen, Switzerland) for $2 \mathrm{~h}$. After preplating, the purified cells were cultured in the $10 \%$ Matrige $^{\circledR}{ }^{\circledR}$-coated 24-well plastic tissue culture plates (Techno Plastic Products).

When the primary myoblasts culture reached $75 \%$ confluency, they were trypsinised and seeded on 8-well removable micro-chamber slip (Ibidi USA, Madison, WI, USA) at a density of 1,000 cells/well in conditioned medium. The primary myoblast cultures were subsequently fixed and subjected to double immunocytochemical staining. In brief, the cells were fixed with $4 \%(\mathrm{w} / \mathrm{v})$ paraformaldehyde $(\mathrm{pH} 7.4)$ in PBS for $10 \mathrm{~min}$, permeabilized with $0.5 \%(\mathrm{v} / \mathrm{v})$ Triton X-100 for $8 \mathrm{~min}$ and blocked with 1X PBS containing $1 \%(\mathrm{v} / \mathrm{v})$ goat serum (Gibco) overnight at $4^{\circ} \mathrm{C}$. The cells were incubated with primary antibody for overnight at $4^{\circ} \mathrm{C}$ then with secondary antibody for $1 \mathrm{~h}$ at room temperature followed by mounting in ProLong ${ }^{\circledR}$ Gold Antifade Reagent (Invitrogen, Carlsbad, CA, USA) containing DAPI. Self-renewing, proliferating, and differentiating myoblast progeny were distinguished as Pax7+MyoD-, Pax7+MyoD+ and Pax7-MyoD+, respectively [17]. Myoblast proliferation and differentiation were confirmed using the cell proliferation marker Ki67 [1ㅇ] combined with the myogenic marker MyoD. Proliferating and differentiating myoblast progeny were distinguished as Ki67+MyoD+ (proliferating) and Ki67-MyoD+ (differentiating). Primary antibodies used were mouse monoclonal anti-Pax7 (1:5; clone Pax7; Developmental Studies Hybridoma Bank, lowa City, IA, USA), mouse monoclonal anti-MyoD (1:200; clone 5.8A; Thermo Fisher Scientific, Inc., MA, USA), and rabbit polyclonal anti-MyoD (1:200; Santa Cruz Biotechnology, Santa Cruz, CA, USA).

Fluorescent microscope images were acquired then processed and assembled into figures using ImageJ (http://rsbweb.nih.gov/ij/). The number of progeny 
from each different group was counted manually in images captured at 20X magnification using ImageJ. The self-renewal, proliferation and differentiation indices of progeny were determined by calculating the mean percentage of Pax7+MyoD- (self-renewing); Pax7+MyoD+ and Ki67+MyoD+ (proliferating) and Pax7MyoD+ and Ki67-MyoD+ (differentiating) progeny in at least 200 cells. Significant differences between Ts1Cje and WT controls were determined using Student's ttest. A minimum of three replicates were analyzed for each experiment.

\section{RESULTS}

\subsection{Assessment of satellite cell populations and} myonuclei number in skeletal muscle of Ts1Cje mice

Associated satellite cells were quantified by Pax 7 immunostaining (a marker for quiescent satellite cells), and all myonuclei were counterstained with DAPI (Figure 1a). The corresponding results showed no significant differences $(P>0.05)$ in the number of Pax7- expressing satellite cells per myofiber between the two genotypes in both male (WT $=7.73 \pm 0.8$; Ts1Cje $=6.97$ $\pm 0.6 ; n=4 ; P=0.5845)$ and female (WT = $4.75 \pm 0.6$; Ts1Cje $=4.76 \pm 0.5 ; n=5 ; P=0.9883$ ) groups, suggesting there are no differences in the satellite cell pools between both genotypes and genders (Figure 1b).

To further investigate the size of myofibers, the average number of myonuclei present in each myofiber was assessed. The results showed there was no significant difference $(P>0.05)$ in the average number of myonuclei present in each myofiber of the two genotypes for both genders (male: WT $=268.70 \pm 9$, Ts1Cje $=253.83 \pm 11, n=4, P=0.4572$; female: $W T=$ $233 \pm 8$, Ts1Cje $=213.59 \pm 8, n=5, P=0.2844$; Figure 1c). These data suggest that Ts1Cje mouse myofibers are similar in size to those of WT mice. Taken together, normal satellite cell pool size and myonuclear populations are present in the Ts1Cje mouse skeletal muscle. a
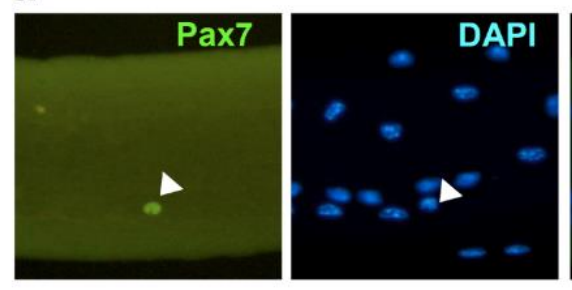

d

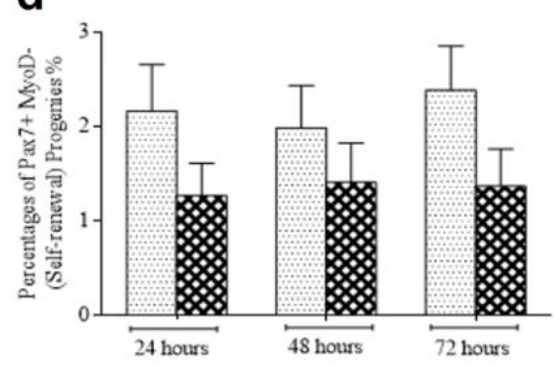

b

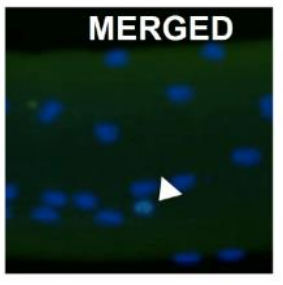

e

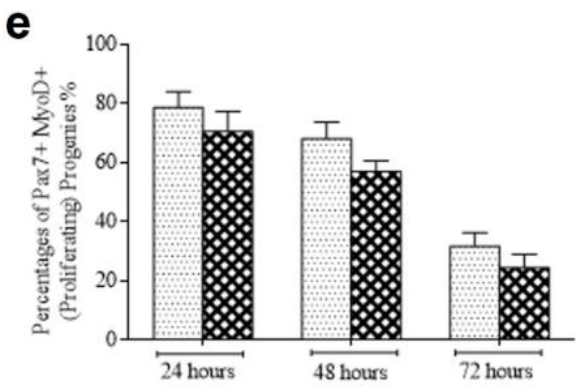

C
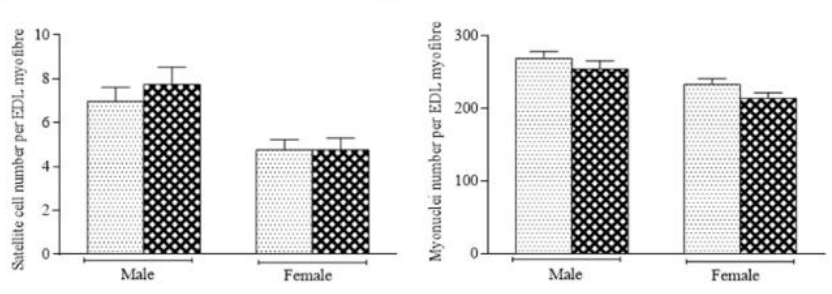

f

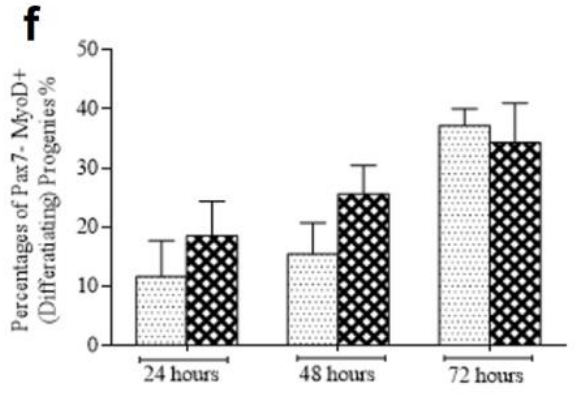

Figure 1. Analysis of the satellite cells and myonuclei in the trisomic Ts1Cje skeletal muscle. (a) Isolated single myofibres immunostained with (i) Pax7 and (ii) DAPI show satellite cell and myonuclei. Scale bar 2000 $\mu$ m. Student t-test revealed both (b) average number of satellite cells and (c) average number of myonuclei in each myofiber did not differ significantly between the two genotypes for both genders. In vitro cellular function analysis also demonstrated that the (d) Pax7+MyoD--selfrenewing, (e) Pax7+MyoD+-proliferating and (f) Pax7-MyoD+-differentiating population of satellite cells were not statistically significant between the two genotypes across the 24,48 and 72 consecutive hours of assessment. 


\subsection{Assessment of the cellular function of satellite}

\section{cells from Ts1Cje mouse skeletal muscle}

The self-renewal efficiency of satellite cells was also assessed in this experiment. The Pax7+MyoD- (selfrenewing) satellite cells of the Ts1Cje culture ( $24 \mathrm{~h}: 1.27$ $\pm 0.34 \%$; 48 h: $1.41 \pm 0.41 \% ; 72$ h: $1.37 \pm 0.40 \%$ ) was consistently lower than the WT control ( 24 h: $2.17 \pm$ $0.49 \%$; 48 h: $1.98 \pm 0.44 \% ; 72$ h: $2.09 \pm 0.41 \%)$, although not statistically significant $(24 \mathrm{~h}: \mathrm{P}=0.2136 ; 48 \mathrm{~h}: \mathrm{P}=$ $0.3977 ; 72 \mathrm{~h}: \mathrm{P}=0.2725$ ) (Figure $1 \mathrm{~d}$ ).

We found that the number of Pax7+MyoD+ (proliferating) satellite cells in the WT control cultures ( 24 h: $78.62 \pm 5.40 \% ; 48$ h: $68.09 \pm 5.63 \% ; 72$ h: $31.68 \pm$ $4.63 \%)$ was consistently higher than the number of Pax7+MyoD+ satellite cells in the Ts1Cje culture $(24 \mathrm{~h}$ : $70.58 \pm 6.84 \%$; 48 h: $57.08 \pm 3.53 \% ; 72$ h: $24.47 \pm 4.46 \%$ ), although no significant differences were found between the two genotypes ( $24 \mathrm{~h}: \mathrm{P}=0.4110 ; 48 \mathrm{~h}: \mathrm{P}=0.1863$; $72 \mathrm{~h}: \mathrm{P}=0.3254$; Figure 1e). These data agreed with that obtained based on MyoD and Ki67 expression patterns, which also demonstrated that there was no difference in proliferative potential of satellite cells between the two genotypes $(24 \mathrm{~h}$ : WT $=$

\section{a}

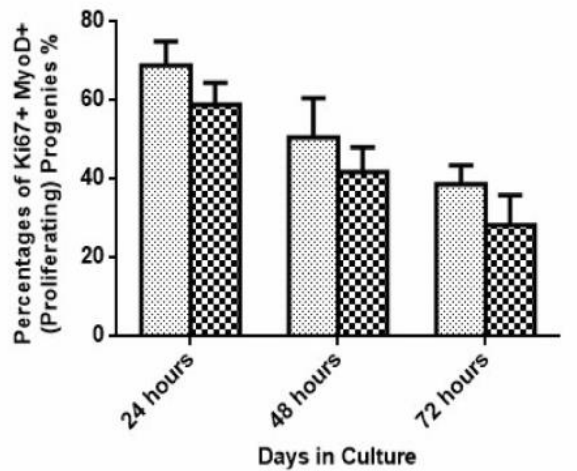

$68.71 \pm 6.14 \%$, Ts1Cje $=58.70 \pm 5.69 \%, P=0.4110 ; 48 \mathrm{~h}:$ $\mathrm{WT}=50.42 \pm 9.98 \%, \mathrm{Ts} 1 \mathrm{Cje}=41.64 \pm 6.30 \%, \mathrm{P}=0.5056$; $72 \mathrm{~h}: \mathrm{WT}=38.54 \pm 4.76 \%, \mathrm{Ts} 1 \mathrm{Cje}=28.11 \pm 7.62 \%, \mathrm{P}=$ 0.3213 ; Figure 2a).

We also further assessed the differentiation potential of satellite cells. Based on the data obtained at 24, 48 and $72 \mathrm{~h}$ after cell seeding. The percentage of Pax7-MyoD+ (differentiating) satellite cells in the Ts1Cje culture (24 h: $18.57 \pm 5.84 \% ; 48$ h: $25.50 \pm 4.74 \% ; 72$ h: $34.30 \pm$ $6.73 \%$ ) was not significantly different to that of the WT control culture $(24 \mathrm{~h}: 11.68 \pm 6.00 \% ; 48 \mathrm{~h}: 15.50 \pm$ 5.28\%; 72 h: $37.20 \pm 2.88 \% ; 24$ h: $\mathrm{P}=0.4595 ; 48$ h: $\mathrm{P}=$ $0.2328 ; 72$ h: $P=0.7213$; Figure 1f). Additionally, similar results were obtained based on MyoD and Ki67 expression patterns analysis, which also suggested that the differentiation potential was not significantly different between the two genotypes across the 3 consecutive days of evaluation ( $24 \mathrm{~h}$ : WT $=16.45 \pm$ $8.47 \%$, Ts1Cje $=23.02 \pm 7.01 \%, P=0.5834) ;(48 \mathrm{~h}: \mathrm{WT}=$ $20.53 \pm 9.43 \%$, Ts1Cje $=31.91 \pm 3.42 \%, P=0.3346) ;(72$ $\mathrm{h}: \mathrm{WT}=21.97 \pm 4.89 \%, \mathrm{Ts} 1 \mathrm{Cje}=19.02 \pm 5.72 \%, \mathrm{P}=$ 0.7158 ; Figure $2 b$ ).

\section{b}

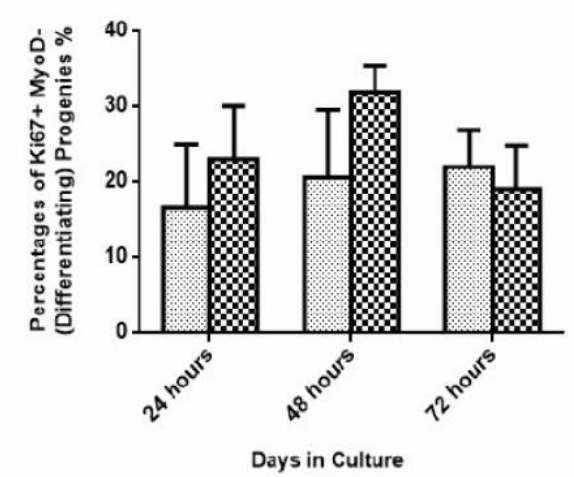

Figure 2. Percentage of proliferating and differentiating progenies of satellite cells. (a) Ki67+MyoD+ proliferating satellite cells showed no significant difference between two genotypes [24 h: WT $=68.71 \pm 6.14 \%$, Ts1Cje $=58.70 \pm 5.69 \%, P=0.2985 ; 48 \mathrm{~h}$ : $\mathrm{WT}=50.42 \pm 9.98 \%, \mathrm{Ts} 1 \mathrm{Cje}=41.64 \pm 6.30 \%, \mathrm{P}=0.5056 ; 72 \mathrm{~h}: \mathrm{WT}=38.54 \pm 4.76 \%, \mathrm{Ts} 1 \mathrm{Cje}=28.11 \pm 7.62 \%, \mathrm{P}=0.3213 \mathrm{]} .(\mathrm{b})$ Ki67+MyoD- differentiating satellite cells of Ts1Cje were not significantly different from WT $[24 \mathrm{~h}:$ WT = 16,45 $\pm 8.47 \%$, Ts1Cje $=23.02 \pm 7.01 \%, P=0.5834 ; 48 \mathrm{~h}: \mathrm{WT}=31.92 \pm 3.42 \%, \mathrm{Ts} 1 \mathrm{CJ}$ e $=57.08 \pm 6.11 \%, \mathrm{P}=0.3346 ; 72 \mathrm{~h}: \mathrm{WT}=21.97 \pm 4.89 \%, \mathrm{Ts} 1 \mathrm{Cje}=$ $19.02 \pm 5.72 \%, P=0.7158]$. 


\section{DISCUSSION}

Our current data shows that both the satellite cell pools and the myonuclear populations present in the Ts1Cje mouse skeletal muscle are similar to those of WT. The myonuclear number often reflects the size of satellite cell populations because satellite cells are the main source of myonuclei during early postnatal muscle development and adult skeletal muscle regeneration [19-20]. As such, the satellite cell number is always directly proportional to the myonuclei number present on myofibers. To the best of our knowledge, this is the first study to profile these muscle cells in a DS model.

The myonuclei number also reveals the size of the myofiber. Based on the myonuclear domain hypothesis, the nucleus-to-cytoplam ratio will remain constant during myofiber growth; hence, any increase in myofiber size must occur via addition of new myonuclei from satellite cell proliferation. In the current study, we found a normal number of myonuclei present in Ts1Cje mouse myofibers, indicating that these myofibers are comparable to that of WT mice. Even though this interpretation was predicted based on the myonuclear domain hypothesis [21-22], it supports data from our recent study on myofiber cross-sections [unpublished data]. In that study, the cell cross-sectional area and morphology of the Ts1Cje mouse skeletal muscle were investigated. The results of that study showed no significant difference in terms of the cell cross-sectional area and morphology between the two genotypes [unpublished data].

The present in vitro analyses revealed no significant differences of the functionality and populations of the satellite cells derived from Ts1Cje mice and WT littermates, indicating that the trisomic genes may exert minimal influence on the self-renewal, proliferation and differentiation profiles of the satellite cells. These results further suggest that the intrinsic cellular functionality of the DS satellite cell is similar to that of the WT. This finding is still less conclusive because the present experiments revealed only the in vitro cellular functionality of satellite cells and not the in vivo skeletal muscle regeneration process. Skeletal muscle regeneration is a highly synchronized process governed by both intrinsic and extrinsic regulatory cues and is also tightly linked to the local and systemic metabolism of a biological system [23-24]. Hence, further studies to reveal the overall in vivo skeletal muscle regeneration process of Ts1Cje mouse skeletal muscle are necessary in the future.

\section{CONCLUSIONS}

Overall, we showed that the DS skeletal muscle has similar satellite cell population and intrinsic cellular functionality as the WT.

Acknowledgements: This work was supported in part by funding from the Fundamental Research Grant Scheme (0401-15-1663FR) awarded to P-SC. C-LL was a recipient of the Malaysian Ministry of Higher Education MyMaster scholarship. Monoclonal antibody, Pax7 was developed by A. Kawakami and obtained from the Developmental Studies Hybridoma Bank developed under the auspices of the NICHD and maintained by the Department of Biological Sciences, at the University of lowa (lowa City, IA, USA).

Author Contributions: All authors of this research paper have directly participated in the planning, execution, or analysis of this study; CLL, K-HL, and P-SC designed the experiments. C$L L, U B$, and MP-YL performed experiments and analyzed the data. IKSY, JS, and RR co-supervised experimentation. CLL, K$\mathrm{HL}$ and $\mathrm{P}-\mathrm{SC}$ drafted the manuscript.

Conflicts of Interest: The authors declare no conflict of interest.

\section{References}

1. Roper R.J., Reeves R.H. Understanding the basis for Down syndrome phenotypes. PLoS Genet 2006, 2: e50. https://doi.org/10.1371/journal.pgen.0020050 
2. National Center on Birth Defects and Developmental Disabilities (NCBDDD) | CDC. National Center on Birth Defects and Developmental Disabilities Home / NCBDDD / CDC. 2017. Available at: http://www.cdc.gov/ncbddd/birthdefects/downsyndrome.html. Accessed May 26, 2017.

3. Brault V., Duchon A., Romestaing C., Sahun I., Pothion S., Karout M., et al. Opposite phenotypes of muscle strength and locomotor function in mouse models of partial trisomy and monosomy 21 for the proximal Hspa13-App region. PLOS Genet 2015, 11: e1005062. https://doi.org/10.1371/journal.pgen.1005062

4. Lisi E.C., Cohn R.D. Genetic evaluation of the pediatric patient with hypotonia: perspective from a hypotonia specialty clinic and review of the literature. Dev Med Child Neurol 2011, 53: 586-599.

https://doi.org/10.1111/j.1469-8749.2011.03918.x

5. Cowley P.M., Keslacy S., Middleton F.A., DeRuisseau L.R., Fernhall B., Kanaley J.A., DeRuisseau K.C. Functional and biochemical characterization of soleus muscle in Down syndrome mice: insight into the muscle dysfunction seen in the human condition. Am J Physiol Regul Integr Comp Physiol 2012, 303: R1251-R1260. https://doi.org/10.1152/ajpregu.00312.2012

6. Yin H., Price F., Rudnicki M. Satellite cells and the muscle stem cell niche. Physiol Rev 2013, 93: 23-67. https://doi.org/10.1152/physrev.00043.2011

7. Boldrin L., Morgan J.E. Human satellite cells: identification on human muscle fibres. PLoS Curr 2013, 3: RRN1294. https://doi.org/10.1371/currents.RRN1294

8. Dumont N.A., Wang Y.X., Rudnicki M.A. Intrinsic and extrinsic mechanisms regulating satellite cell function. Development 2015, 142: 1572-1581. https://doi.org/10.1242/dev.114223

9. Tierney M.T., Sacco A. The role of muscle stem cell-niche interactions during aging. Nat Med 2016, 22: 837838. https://doi.org/10.1038/nm.4159

10. Shefer G., Van de Mark D.P., Richardson J.B., Yablonka-Reuveni Z. Satellite-cell pool size does matter: Defining the myogenic potency of aging skeletal muscle. Dev Biol 2006, 294: 50-66.

https://doi.org/10.1016/j.ydbio.2006.02.022

11. Shefer G., Rauner G., Yablonka-Reuveni Z., Benayahu D. Reduced satellite cell numbers and myogenic capacity in aging can be alleviated by endurance exercise. PLOS ONE 2010, 5: e13307.

https://doi.org/10.1371/journal.pone.0013307

12. Sago H., Carlson E., Smith D., Kilbridge J., Rubin E., Mobley W.C., Epstein C.J., Huang T.T. Ts1Cje, a partial trisomy 16 mouse model for Down syndrome, exhibits learning and behavioral abnormalities. Proc Natl Acad Sci USA 1998, 95: 6256-6261. https://www.ncbi.nlm.nih.gov/pubmed/9600952

13. Liu B., Filippi S., Roy A., Roberts I. Stem and progenitor cell dysfunction in human trisomies. EMBO Rep 2015, 16: 44-62. https://doi.org/10.15252/embr.201439583

14. Shimohata A., Ishihara K., Hattori S., Miyamoto H., Morishita H., Ornthanalai G., Raveau M., Ebrahim A.S., Amano K., Yamada K., Sago H., Akiba S., Mataga N., Murphy N.P., Miyakawa T., Yamakawa K. Ts1Cje Down syndrome model mice exhibit environmental stimuli-triggered locomotor hyperactivity and sociability concurrent with increased flux through central dopamine and serotonin metabolism. Exp Neurol. 2017, 293: 1-12. https://doi.org/10.1016/j.expneurol.2017.03.009

15. Ling K.H., Hewitt C.A., Tan K.L., Cheah P.S., Vidyadaran S., Lai M.I., et al. Functional transcriptome analysis of the postnatal brain of the Ts1Cje mouse model for Down syndrome reveals global disruption of interferonrelated molecular networks. BMC Genomics 2014, 15: 624. https://doi.org/10.1186/1471-2164-15-624 
16. Lim C.L., Ling K.H., Cheah P.S. Isolation, cultivation and immunostaining of single myofibers- an improved approach to study the behaviour of satellite cells. J Biol Methods 2018, 5: e87. https://doi.org/10.14440/jbm.2018.219

17. Zammit P.S., Golding J.P., Nagata Y., Hudon V., Partridge T.A., Beauchamp J.R. Muscle satellite cells adopt divergent fates. J Cell Bio/ 2004, 166:347-357. https://doi.org/10.1083/icb.200312007

18. Schlüter C., Duchrow M., Wohlenberg C., Becker M.H., Key G., Flad H.D., et al. The cell proliferationassociated antigen of antibody Ki-67: a very large, ubiquitous nuclear protein with numerous repeated elements, representing a new kind of cell cycle-maintaining proteins. J Cell Biol 1993, 123: 513-522. https://doi.org/10.1083/jcb.123.3.513

19. Allen D.L., Roy R.R., Edgerton V.R. Myonuclear domains in muscle adaptation and disease. Muscle Nerve 1999, 22: 1350-1360. https://www.ncbi.nlm.nih.gov/pubmed/10487900

20. Brack A.S. Evidence that satellite cell decrement contributes to preferential decline in nuclear number from large fibres during murine age-related muscle atrophy. J Cell Sci 2005, 118: 4813-4821. https://doi.org/10.1242/jcs.02602

21. Van der Meer S.F., Jaspers R.T., Degens H. Is the myonuclear domain size fixed? J Musculoskelet Neuronal Interact 2011, 11: 286-297. https://www.ncbi.nlm.nih.gov/pubmed/22130137

22. Neal A., Boldrin L., Morgan J.E. The satellite cell in male and female, developing and adult mouse muscle: distinct stem cells for growth and regeneration. PLOS ONE 2012, 7: e37950.

https://doi.org/10.1371/journal.pone.0037950

23. Koopman R., Ly C.H., Ryall J.G. A metabolic link to skeletal muscle wasting and regeneration. Front Physiol 2014, 5: 32. https://doi.org/10.3389/fphys.2014.00032

24. Jang Y.C., Sinha M., Cerletti M., Dall'Osso C., Wagers A.J. Skeletal muscle stem cells: effects of aging and metabolism on muscle regenerative function. Cold Spring Harb Symp Quant Biol 2011, 76: 101-111. https://doi.org/10.1101/sqb.2011.76.010652 